\title{
Grid laser photocoagulation for macular oedema in uveitis or the Irvine-Gass syndrome
}

Department of Ophthalmology, FC Donders Institute, University Hospital Utrecht, Utrecht, Netherlands C W T A Lardenoye $M \mathrm{~J}$ van Schooneveld W Frits Treffers

A Rothova

Correspondence to: Charlotte W T A Lardenoye, Department of

Ophthalmology, FC Donders Institute, University Hospital

Utrecht, PO Box 85500 3508 GA Utrecht,

Netherlands.

Accepted for publication 17 March 1998

\author{
Charlotte W T A Lardenoye, Mary J van Schooneveld, W Frits Treffers, Aniki Rothova
}

\begin{abstract}
Aims-To determine the effect of modified macular grid photocoagulation in patients with refractory macular oedema due to uveitis or cataract extraction.

Methods-In this study 20 patients with macular oedema underwent modified macular grid laser photocoagulation and were followed by means of standardised examinations (day 0 , months 2,6 , and 12) consisting of best corrected visual acuity and fluorescein angiography.

Results-The mean visual acuity increased from 0.16 before to 0.3 after laser treatment $(p=0.013)$, and fluorescein leakage was significantly reduced $(p=0.005)$. Visual prognosis was influenced by duration of the uveitis, not by sex or age. Conclusion-Modified macular grid laser photocoagulation had a beneficial effect on macular oedema caused by uveitis or the Irvine-Gass syndrome. A prospective, randomised study is needed to determine the exact place of modified macular grid photocoagulation in the treatment of patients with inflammatory or postsurgical macular oedema.
\end{abstract}

(Br f Ophthalmol 1998;82:1013-1016)

Macular oedema is a major complication of various diseases, including uveitis, diabetes mellitus, retinal vascular diseases, retinitis pigmentosa, and radiation retinopathy as well as cataract surgery (Irvine-Gass syndrome). Treatment of macular oedema includes nonsteroidal anti-inflammatory drugs, corticosteroids, other immunosuppressive agents, and acetazolamide, but resistant cases of macular oedema are common. ${ }^{1-3}$ Beneficial results of vitrectomy and hyperbaric oxygen treatment have been described in small series of patients. ${ }^{45}$

The macular grid laser had a positive effect on visual acuity and fluorescein leakage in patients with diabetic macular oedema and those with radiation retinopathy. ${ }^{67}$ To our knowledge, only one study addresses the effect of the macular grid laser in uveitis. In this study, five patients with refractory cystoid macular oedema of long duration and poor visual acuity exhibited a reduction in oedema but no significant increase in visual acuity, ${ }^{8}$ findings similar to those obtained with grid laser photocoagulation after central vein occlusion. $^{9}$

The main purpose of this study was to investigate whether grid laser photocoagulation has a beneficial effect in patients with inflammatory or postsurgical macular oedema.

\section{Materials and methods}

Twenty patients who were older than 18 years had macular oedema, 14 with chronic uveitis, and six with the Irvine-Gass syndrome were enrolled in this study. All patients with uveitis underwent standardised screening to detect the aetiology of their ocular disease. Clinical diagnosis included sarcoid associated uveitis $(n=4)$, presumed ocular borreliosis $(n=1)$, acute retinal necrosis $(n=1)$, and eight cases of unknown origin. None of the patients had diabetes mellitus.

Inclusion criteria were visual acuity less than 20/40 due to refractory macular oedema lasting for at least 6 months. For patients with bilateral oedema, the worse eye was eligible for grid treatment. The male:female ratio was 1:6. The average age of the whole group at the time of photocoagulation was 60 years (range 32-83), 70 years for the Irvine-Gass group, and 55 years for those with uveitis. The mean duration of macular oedema before laser treatment was 3 years (range 6 months to 15 years). At the beginning of the study, the degree of inflammation for all eyes to be treated and for the control group was equal to or less than grade $1 .^{10}$ The systemic treatment was concurrently used in patients and the administration and dosages remained unchanged during the study (four with systemic corticosteroids, one with cyclosporin, and six with acetazolamide).

For additional comparison of changes in visual acuity (day 0, months 2 and 6 ) historical controls were selected, consisting of 14 consecutive sex and age matched patients with uveitis, and six sex and age matched patients who had undergone cataract extraction. All controls had macular oedema as confirmed by fluorescein angiography with an average duration of 3 years (range 6 months to 8 years) and had received conventional treatment. The inflammatory activity and conventional treatment used during the observation period did not differ between the treated patients and the controls (controls: two with systemic corticosteroids, one with cyclosporin, and seven with acetazolamide). During the course of the study, two patients (one of the treated group and one of the control group) experienced reactivation of uveitis (the increase in macular oedema was treated with a periocular triamcinolone injection and acetazolamide, respectively).

The patients underwent modified macular grid photocoagulation with a yellow dye laser (wavelength $577 \mathrm{NM}$ ). The spot size was 100$200 \mu \mathrm{m}$, duration 0.07-0.15 seconds, and initial low intensity was gradually increased until the coagulate was just visible. A pattern of 20-40 spots was placed throughout the areas of 
macular oedema. The central area (up to 500 $\mu \mathrm{m}$ from the centre of the fixation point) and non-oedematous retina were not treated. All laser treatments were performed by the same ophthalmologist (AR).

Standardised examinations were performed before and 2, 6, and 12 months after grid laser photocoagulation. Best corrected visual acuity was determined with the Snellen letter projector in a uniform manner and always in the same examination environment. For statistical analysis of visual acuity the $\log$ MAR visual acuity (logarithm of the minimal angle of resolution) was used. The diagnosis of macular oedema was based on both clinical criteria and fluorescein angiography. Fluorescein angiography was performed in all cases before laser treatment and in all but two cases 6 months later. Late phase fluorescein angiograms were graded by two masked observers to analyse the area of the macula affected by oedema. Patients with no leakage on the late phase angiogram were graded 0 , those with less than $25 \%$ of the macular area affected by oedema as grade 1, those with leakage between $25 \%$ and $66 \%$ (two thirds) as grade 2 , and those with severe leakage of more than $66 \%$ as grade 3 . A foveal avascular zone was considered abnormal when its size was larger than 0.5 disc diameter or when its border was grossly disrupted. ${ }^{11}$

The study protocol was approved by the committee for scientific research in humans of our hospital, and informed consent was obtained from participating patients. For statistical analysis we used the Wilcoxon signed ranks test and the Student's $t$ test. We also used repeated measurement analysis of variance to determine whether age, sex, duration of uveitis and Irvine-Gass, and duration of macular oedema correlated statistically with visual gain. A p value of 0.05 or less was considered indicative of statistical significance.

\section{Results}

Mean pretreatment visual acuity for the entire series (uveitis and the Irvine-Gass syndrome) was 0.16 . Two months after laser treatment we found an increase in the mean visual acuity to 0.3 , which persisted during 12 months of follow up (Figs 1 and 2). The prognosis for visual acuity was not associated with pretreatment visual acuity: for four out of seven eyes with a pretreatment vision of 0.2 or less, an increase in visual acuity of one Snellen line or more was achieved. The difference between the

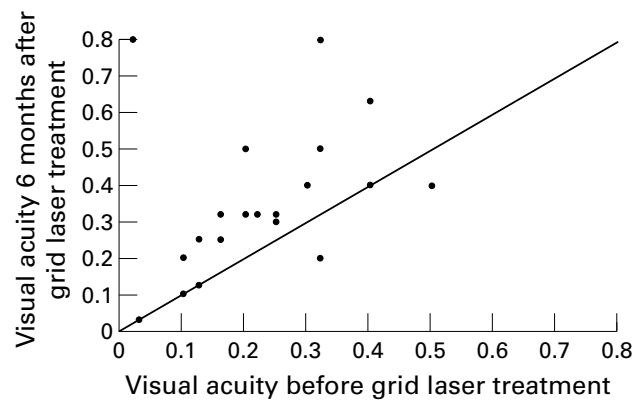

Figure 1 Visual acuity of treated patients before compared with 6 months after grid laser treatment.

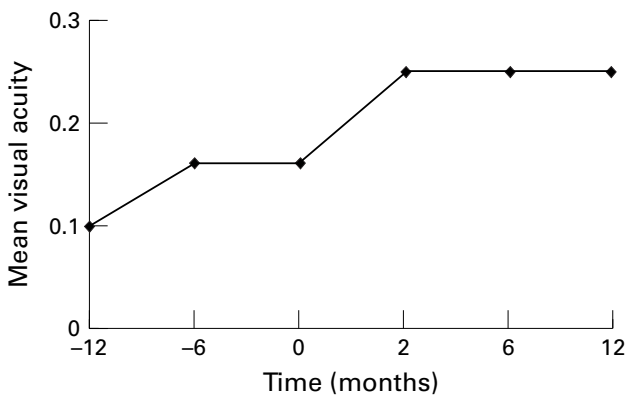

Figure 2 Mean visual acuity 1 year and 6 months before grid laser treatment, at the day of laser treatment, and 2, 6 , and 12 months after grid laser treatment.

visual acuity before and after laser treatment was significant $(2$ months $p=0.02 ; 6$ months $\mathrm{p}=0.01$ ), in contrast with the control group which exhibited no significant visual gain (uveitis controls, $\mathrm{p}=0.6$, Irvine-Gass controls; $\mathrm{p}=0.9)$. At 6 months, visual acuity has increased two Snellen lines or more for 10/20 treated eyes in contrast with $3 / 20$ controls $(\mathrm{p}=0.02)$.

The area of fluorescein leakage on the angiogram was significantly reduced after grid laser treatment $(\mathrm{p}=0.005$; Figs 3 and 4$)$. The pretreatment amount of fluorescein leakage did not influence the visual prognosis; visual acuity increased after laser treatment for seven out of 11 patients with fluorescein leakage grade 3 (mean increase in this group 2.1 Snellen lines). Seven out of 12 patients with improvement in visual acuity $(58.3 \%)$ also exhibited decrease of leakage on fluorescein angiography (in five remaining patients the leakage remained unchanged). For all five patients with disrupted or enlarged foveal avascular zone the visual acuity increased (mean increase 2.4 Snellen lines).

Repeated measurement analysis of variance revealed a significant influence of the duration of uveitis or Irvine-Gass syndrome $(p=0.001)$ on the visual prognosis. Statistical analysis did not reveal any significant correlation between visual outcome and sex or age of the patients or the duration of macular oedema. Nevertheless, when we divided the treated patients into two groups, one with macular oedema lasting 2 years or less and the other with a duration exceeding 2 years (mean visual acuity 0.2 and 0.125 , respectively; $p=0.13$ ), the follow up visual acuity for the group with macular oedema of longer duration was significantly worse (mean visual acuity 0.4 and 0.16 , respectively; $p=0.03)$. Of the five patients with a final visual acuity of $20 / 40$ or more at the last follow up four were younger than 60 years.

The improvement of visual acuity and fluorescein leakage was similar for patients with uveitis and those with the Irvine-Gass syndrome. No treatment related complications (that is, enlargement of laser induced scars, subretinal neovascularisation, or visual decrease of more than one Snellen line) were observed at the 12 month follow up.

\section{Discussion}

Our study revealed a beneficial effect of grid laser photocoagulation on both visual acuity 

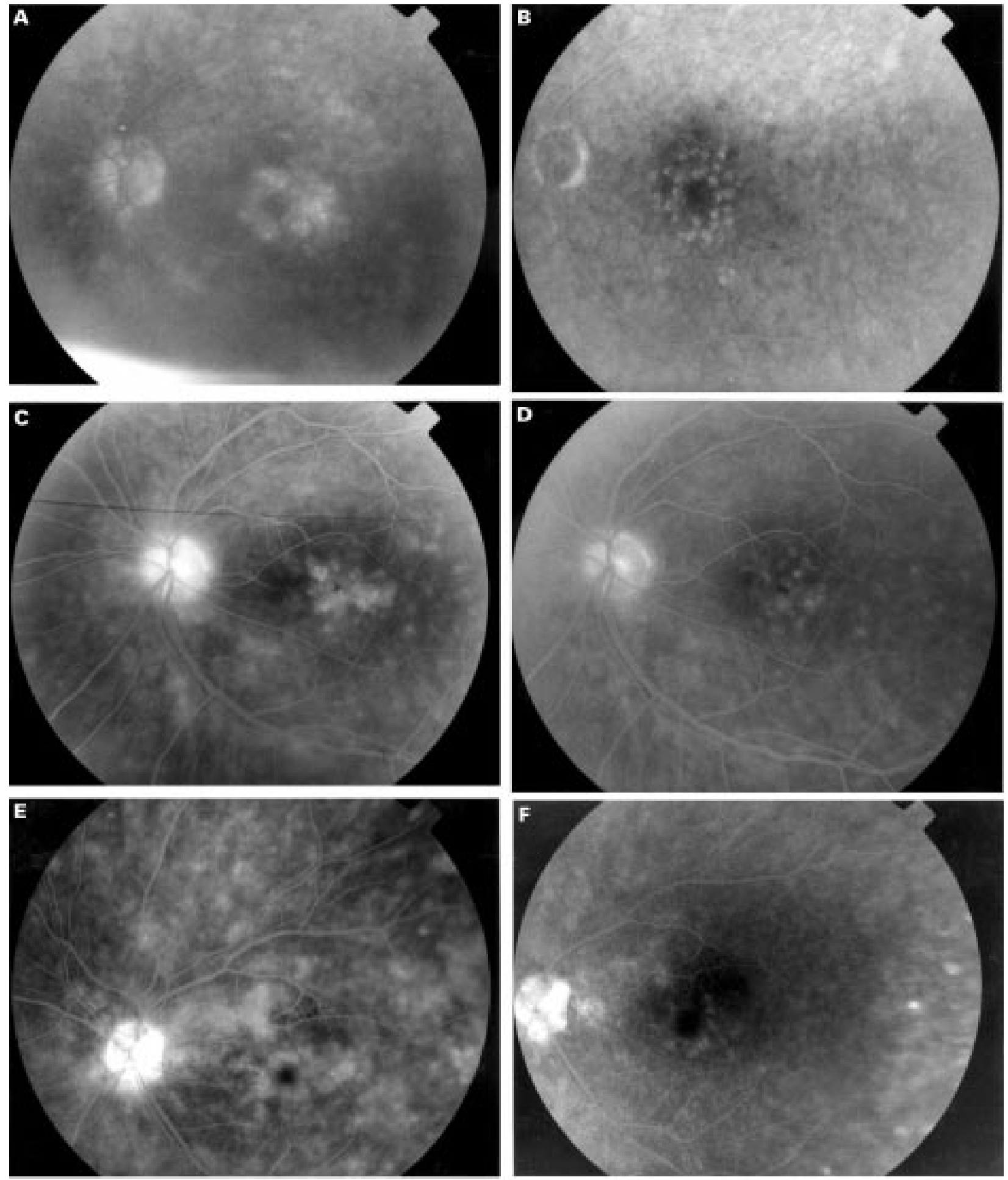

Figure 3 Fluorescein angiography of three cases. Left $(A, C$, and $E)$; before grid laser treatment; right $(B, D$, and $F)$ the same eyes 6 months after grid laser treatment.

and fluorescein leakage in inflammatory and postsurgical macular oedema which did not respond to conventional treatment. This effect was already present after a follow up period of 2 months and persisted for at least 1 year. The improvement in visual acuity and angiographic findings was not observed for control patients. The use of historical controls (despite the fact that they were matched for sex, age, and inflammatory activity) has serious limitations; however, the beneficial effect of the grid laser was obvious as illustrated by the changes and the onset of improvement in visual acuity in treated patients (Fig 2).

The mechanisms by which laser photocoagulation prevents visual loss due to macular oedema are not yet completely understood. The therapeutic benefit of retinal photocoagu- 


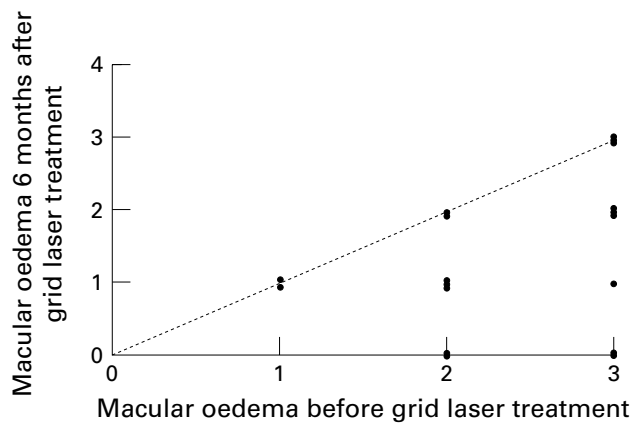

Figure 4 Angiographic macular oedema before compared with 6 months after grid laser treatment (for grading see Materials and methods). Cases under the line represent eyes with decreased leakage on follow up angiogram.

lation could be caused by the tissue response induced by photocoagulation. Either removal of the oxygen consuming photoreceptors and replacement by low oxygen consuming glial scar or regeneration of the retinal pigment epithelium (RPE), or endothelial cell renewal mediated by growth factors which reach the capillaries via the altered RPE might cause the therapeutic effect. ${ }^{6-12}$ Others postulated that laser treatment of patients with diabetic macular oedema leads to an increased oxygen flux from the choroid to the inner retina, resulting in an autoregulatory vasoconstriction of resistance vessels (arterioles). ${ }^{13}$ This vasoconstriction leads to a decreased intravascular and transmural hydrostatic pressure in the capillaries and venules and eventually to decreased retinal oedema. ${ }^{13}$ However, the commonly accepted explanation is that debridement of the RPE by laser light induces regeneration of new RPE cells with improved functional capacities. It is not known how long the effect of the grid laser will last, especially when the cause of the macular oedema has not been eliminated. The long term visual outcome for patients with uveitis is undoubtedly influenced by the future activity of the inflammatory process. The question of whether the laser treated macula enjoys extra protection in the event of reactivation of the uveitis has not been answered and may only be elucidated by long term follow up.

For patients with diabetes mellitus the grid laser appears to be able to maintain or improve visual acuity, especially for "early treated" eyes. ${ }^{14}$ Our series also showed a better visual prognosis for patients with macular oedema of short duration (less than 2 years). In this series, the identification of prognostic factors revealed that duration of uveitis was an important factor for the visual prognosis, whereas no associa- tions were found for the sex and age of the patients. For patients with macular oedema due to central vein occlusion, older age, macular ischaemia, a foveal avascular zone larger than 0.5 disc diameter and pigmentary clumps in the foveal area were associated with a poor visual prognosis. ${ }^{11}$ Nevertheless, in our study disrupted foveal avascular zone was not associated with a poor prognosis, since all five patients with disruption of the foveal avascular zone experienced improved visual acuity after macular grid photocoagulation.

On the basis of this non-randomised study, we conclude that macular grid photocoagulation may be a valuable alternative in cases of refractory macular oedema. The indications for this treatment are as yet unknown. Therefore, an evaluation of the efficacy of grid laser treatment, preferably by means of a prospective, randomised study, is needed to determine its therapeutic value for macular oedema of inflammatory origin.

The authors thank T T J M Berendschot, $\mathrm{PhD}$, for his advice on statistical analysis.

Supported by Dr F P Fisher Foundation, Mr Klaasstraat 11, 3984 JR Odijk, Netherlands.

1 Dick AD. The treatment of chronic uveitic macular oedema. Br f Ophthalmol 1994;78:1-2.

2 Cox SN, Hay E, Bird AC. Treatment of chronic macular edema with acetazolamide. Arch Ophthalmol 1988;106: edema with

3 Jampol LM. Pharmacologic therapy of aphakic and pseudoJampol LM. Pharmacologic therapy of aphakic and pseudo-
phakic cystoid macular edema: 1985 update. Ophthalmolphakic cystoid macula
ogy $1985 ; 103: 1134-5$.

ogy 1985; 103:1134-5.
4 Pfoff DS, Thom SR. Preliminary report on the effect of hyperbaric oxygen on cystoid macular edema. 7 Cataract Refract Surg 1987;13:136-40.

5 Dugel PU, Rao NA, Ozler S, et al. Pars plana vitrectomy for intraocular inflammation-related cystoid macular edema unresponsive to corticosteroids. Ophthalmology 1992;99: 1535-41.

6 Lee CM, Olk RJ. Modified grid laser photocoagulation for diffuse diabetic macular edema. Long-term visual results. Ophthalmology 1991;98:1594-602.

7 Kinyoun JL, Zamber RW, Lawrence BS, et al. Photocoagulation treatment for clinically significant radiation macular oedema. Br f Ophthalmol 1995;79:144-9.

8 Suttorp-Schulten MSA, Feron E, Postema F, et al. Macular grid photocoagulation in uveitis. Br f Ophthalmol 1995; 79. grid ph.

9 The Central Vein Occlusion Study Group. Evaluation of grid pattern photocoagulation for macular edema in central vein occlusion. The Central Vein Occlusion Study Group M Report. Ophthalmology 1995;102:1425-33.

10 Nussenblatt RB, Whitcup SM, Palestine AG. Examination of the patient with uveitis. In: Nussenblatt RB, Whitcup SM, Palestine AG, eds. Uveitis. Fundamentals and clinical practice. 2nd ed. Chicago: Year Book Medical Publishers, 1996:58-68

11 Glacet BA, Mahdavi KN, Coscas G, et al. Macular grid photocoagulation in persistent macular edema due to central retinal vein occlusion. Eur f Ophthalmol 1994;4:16674.

12 Roider J, Michaud NA, Flotte TJ, et al. Response of the retinal pigment epithelium to selective photocoagulation. Arch Ophthalmol 1992;110:1786-92.

13 Gottfredsdóttir MS, Stefánsson E, Jónasson F, et al. Retinal vasoconstriction after laser treatment for diabetic macular vasoconstriction after laser treatment for

14 Olk RJ. Modified grid argon (blue-green) laser photocoagulation for diffuse diabetic macular edema. Ophthalmology 1986;93:938-50. 